\title{
Inhalt des Heftes 3 des 1. Bandes
}

Selte

Hzinze, G., Zytoarchitektonische Untergliederung der Area occipitalis . . . . . 173

Hzınze, G., Größenbestimmung und Darstellung von Rindenfeldern . . . . . . . . 199

Olszewski, J., Uber die Gestaltung der Randkörperchen der Nucleoli einiger menschlicher Nervenzellarten . . . . . . . . . . . . . . . . . . . 206

Hopf, A., Die Myeloarchitektonik des Isokortex temporalis beim Menschen . . . . . 208

$\mathrm{D}$ as „Journal für Hirnforschung" wird, - wie bis 1942 das "Journal für Psychologie und Neurologie" - die Forschungsergebnisse des Institutes für Hirnforschung und allgemeine Biologie in Neustadt/Schwarzwald veröffentlichen. Im Mittelpunkt der Forschungen dieses Institutes steht die Hirnanatomie, und zwar jene Teile derselben, die die wichtigsten Erkenntnisquellen für die räumlichen Beziehungen zwischen materiellem Hirngeschehen und Bewußtseinserscheinungen darstellen. Vertiefung der architektonischen Gliederung des Gehirns, Aufdeckung des anatomischen Ausdrucks individueller Besonderheiten Gesunder, Kranker und „zurechnungsfähiger" Asozialer, Ausnutzung der pathologischen Anatomie für die Schaffung einer ätiologischen Klassifikation der sogenannten funktionellen Neurosen und Psychosen, Klärung der aufbauenden und reparatorischen Funktionen des metamitotischen Arbeitskernes der Nervenzellen: das sind gegenwärtig die Hauptforschungsgebiete des Institutes.

\section{Bestellungen an eine Buchhandlung erbeten}

Wenn Sie unsere Literatur nicht in Ihrer Buchhandlung erhalten können oder Schwierigkeiten bei der Beschaffung haben, dann wenden Sie sich bitte an eine der nachstehenden Auslieferungsstellen oder direkt an den Verlag.

\section{Auslleferung für die Deutsche Demokratische Republik: \\ LKG Leipziger Kommissions- und Großbuchhandel Leipzig C 1 , Leninstraße 16 \\ Auslleferung für die Bundesrepublik: \\ Buchhandlung Kunst und Wissen, Inhaber: Erich Bieber Stuttgart N, Eduard-Pfeiffer-Straße 99 a}

Auslieferung für das gesamte Ausland: Deutscher Buch-Export und -Import, GmbH, Leipzig C 1, Postschließfach 276

\author{
Akademie-Verlag, \\ Berlin W 8, MohrenstraBe 39, Ruf 200386 Sammelnummer \\ Telegramm-Adresse: Akademieverlag Berlin
}

\begin{abstract}
Herausgeber und verantwortlich far den Inhalt: Dr. Cécile und Prof. Oskar Vogt, Institut fär Hirnforschung und allgemeine Biologie, Neustadt/Schwarzwald. Verlag: Akademie-Verlag OmbH., Berlin W 8, Mohrenstra Be 39 (Fernruf: 2003 86); Postscheckkonto: Berlin 350 21. Bestell- und Verlagsnummer dleses Heftes: 1018/1/3. Das "Journal fâr Hirnforschung" erscheint in zwanglosen Heften von verschiedenem Umfang. 6 Hefte bilden einen Band. Preis Je Heft 12,- DM. Ein Band 72,- DM. Satz und Druck: Druckerei „Magnus Poser", Zwelgbetrieb Jena. Veroffentlicht unter der Lizenznummer 1230 des Amtes für Literatur und Verlagawesen der Deutschen Demokratischen Republik. Printed in Germany.
\end{abstract}

